\title{
O consumo de produtos ultraprocessados está associado ao melhor nível socioecocômico das famílias das crianças
}

\author{
The consumption of ultra-processed products is associated with \\ the best socioeconomic level of the children's families
}

\author{
Mariane Alves Silva (https://orcid.org/0000-0002-6518-6534) ${ }^{1}$ \\ Luana Cupertino Milagres (https://orcid.org/0000-0002-3186-7577) ${ }^{1}$ \\ Ana Paula Pereira Castro (https://orcid.org/0000-0002-0017-6626) ${ }^{1}$ \\ Mariana De Santis Filgueiras (https://orcid.org/0000-0003-1932-6126) ${ }^{1}$ \\ Naruna Pereira Rocha (https://orcid.org/0000-0001-7513-3906) ${ }^{1}$ \\ Helen Hermana Miranda Hermsdorff (https://orcid.org/0000-0002-4441-6572) ${ }^{1}$ \\ Giana Zarbato Longo (https://orcid.org/0000-0001-7666-5007) ${ }^{1}$ \\ Juliana Farias Novaes (https://orcid.org/0000-0003-3616-5096) ${ }^{1}$
}

${ }^{1}$ Departamento de Nutrição

e Saúde, Universidade

Federal de Viçosa (UFV).

Av. Peter Rolfs s/n, Campus

Universitário. 36570-

900 Viçosa MG Brasil.

mamariane33@

yahoo.com.br

\begin{abstract}
The aim of this study was to evaluate the intake of ultra-processed foods and associated factors in prepubertal children. It is a cross-sectional study with 378 children aged 8 and 9 years enrolled in public and private schools in Viçosa-MG. Food intake was assessed by three 24-hour dietary recalls. Dietary data were entered into the Diet Pro ${ }^{\circledR}$ 5i software to quantify energy intake. The Two-Step Cluster technique was used to analyze food consumption groups, with the Stata 13 software package. The foods were grouped and classified as "healthy" and "unhealthy" eating markers. The association between the sociodemographic variables and the groups formed was examined by Poisson Regression. Two food groups were formed: "healthy" and "unhealthy". The caloric intake of ultra-processed foods was lower in the "healthy" group (20.5\%) than in the "unhealthy" group (24.1\%; $P=0.043)$. The multivariate model showed that private school children $(P R=1.25, P<0.001)$, who did not receive Bolsa Familia $(P R=1.13$, $P=0.036)$ and had working mothers $(P R=$ $1.38, P<0.001)$ had increased probability of unhealthy food consumption. Ultra-processed food intake was associated with greater purchasing power of families of prepubertal children.

Key words Child, Processed foods, Socioeconomic factors.
\end{abstract}

Resumo O objetivo deste artigo é avaliar o consumo de produtos ultraprocessados e fatores associados em crianças pré-púberes. Estudo transversal realizado com 378 crianças de 8 e 9 anos matriculadas em escolas públicas e privadas de Viçosa-MG. O consumo alimentar foi avaliado por três recordatórios de 24h. Os dados dietéticos foram tabulados no software Diet Pro ${ }^{\circledR} 5$ i, para quantificar o consumo energético. Para análise dos grupos de consumo alimentar foi utilizada a técnica Two-Step Cluster, por meio do software Stata versão 13.0. Os alimentos foram agrupados e classificados como marcadores de alimentação "saudável" e "não saudável". A associação entre as variáveis sociodemográficas e os grupos formados foi verificada por meio da Regressão de Poisson. Obteve-se a formação de dois grupos alimentares: "saudável" e "não saudável". A ingestão calórica de ultraprocessados foi menor no grupo "saudável" (20,5\%) em relação ao "não saudável" (24,1\%; $P=0,043)$. No modelo multivariado, crianças de escola privada $(R P=1,25, P<0,001)$, que não recebiam Bolsa Família $(R P=1,13, P=0,036)$ e cuja mãe trabalhava $(R P=1,38, P<0,001)$ apresentaram maior chance de consumo "não saudável". O consumo de produtos ultraprocessados associou-se ao maior poder aquisitivo das famílias de crianças pré-púberes.

Palavras-chave Criança, Alimentos industrializados, Fatores socioeconômicos. 


\section{Introdução}

Nas últimas décadas ocorreram mudanças no padrão alimentar da população brasileira, caracterizado pela diminuição do consumo de alimentos in natura e minimamente processados, em detrimento do aumento da ingestão de produtos processados e ultraprocessados ${ }^{1,2}$. Tais mudanças repercutem numa dieta com maior densidade energética, associado a um aumento na ingestão de aditivos químicos, açúcar, sódio, gordura saturada e trans e à diminuição do consumo de fibras ${ }^{2,3}$.

Os produtos ultraprocessados (UPP) referem-se a formulações da indústria, feitos a partir de substâncias derivadas de alimentos ${ }^{3,4} . \mathrm{Na}$ Pesquisa Nacional de Saúde do Escolar (PeNSE), observou-se um aumento na ingestão de alimentos não saudáveis, como frituras, embutidos, guloseimas e refrigerantes 5 . Esse aumento pode ser atribuído ao ambiente em que o escolar está inserido, pois a maior parte das propagandas de alimentos veiculadas nos meios de comunicação refere-se a produtos industrializados ${ }^{2}$.

Embora ainda sejam escassos estudos que avaliam o consumo individual dos produtos UPP, sabe-se que a disponibilidade domiciliar dos mesmos aumentou junto à prevalência do excesso de peso ${ }^{6}$. Segundo dados da Pesquisa de Orçamento Familiar (POF 2008-2009), 14\% das crianças brasileiras de cinco a nove anos apresentaram obesidade e $33,5 \%$ excesso de peso ${ }^{7}$. Esse cenário se mostra preocupante, uma vez que o estado nutricional e o padrão alimentar adquirido na infância tendem a permanecer na vida adulta $^{8}$.

Fatores sociodemográficos, como a renda e escolaridade dos pais podem estar associados ao consumo de ultraprocessados, no entanto, os estudos são conflitantes quanto a essas associações. Alguns estudos encontraram associação entre o maior consumo de UPP e pior qualidade da dieta com a menor renda e escolaridade dos indivíduos $^{9-11}$, ao passo que outros estudos demonstram maior consumo de UPP conforme aumenta a renda e a escolaridade ${ }^{12-14}$. Diante do exposto, este trabalho objetivou avaliar o consumo de produtos ultraprocessados e os fatores associados em crianças pré-púberes.

\section{Métodos}

\section{População e desenho do estudo}

Trata-se de um estudo quantitativo, descritivo e transversal com amostra representativa de 378 crianças de 8 e 9 anos, matriculadas em escolas urbanas, públicas e privadas do município de Viçosa-MG. Os participantes deste estudo foram provenientes da Pesquisa de Avaliação da Saúde do Escolar (PASE), investigação transversal de base populacional com o objetivo de investigar a saúde cardiovascular deste público infantil no município de Viçosa, MG, Brasil.

O município de Viçosa está localizado na Zona da Mata Mineira e possui uma extensão territorial de $299 \mathrm{~km}^{2}$ e 72.244 habitantes, sendo que $93,2 \%$ residem em zona urbana ${ }^{7}$. Em 2015, o município contava com 24 escolas públicas e privadas urbanas que atendiam 1.464 crianças na faixa etária de 8 e 9 anos.

Para o cálculo amostral foi utilizado o software Epi Info (versão 7.2; Atlanta, GA), a partir da população total de estudantes com 8 e 9 anos segundo dados coletados nas escolas urbanas em 2014/2015. Considerou-se para a realização do cálculo, a população total de estudantes $(\mathrm{n}=1464$ alunos); prevalência de $50 \%$ visto que o estudo considerou desfechos múltiplos; precisão desejada de 5\%; nível de confiança de 95\% e acréscimo de $20 \%$ de perdas ${ }^{15}$, totalizando 366 crianças. Em seguida, considerando a proporção numérica de cada escola, o número de crianças a ser amostrado foi proporcional ao total de alunos existentes em cada uma. Os alunos foram selecionados de forma aleatória, até completar o número de alunos necessários para cada escola.

Não foram incluídas crianças em uso de medicamentos que pudessem alterar o estado nutricional, composição corporal, perfil lipídico, pressão arterial e/ou metabolismo glicídico, com incapacidade física para realizar as medidas antropométricas e com distúrbios do trato gastrointestinal ou orofaríngeo que acarretassem alterações do consumo alimentar. Foi realizado um estudo piloto com 39 crianças de 8 e 9 anos, correspondendo a $10 \%$ da amostra. Estas foram selecionadas de forma aleatória, a fim de testar a aplicação de questionários e de inquéritos alimentares. As crianças selecionadas para o estudo piloto não foram incluídas na amostra final. 


\section{Condições socioeconômicas e demográficas das famílias}

Durante entrevista com os pais ou responsáveis pela criança, foi aplicado um questionário semiestruturado por um nutricionista contendo questões referentes à situação socioeconômica $\mathrm{e}$ ambiental, tais como etnia, renda, escolaridade, participação em programas assistenciais, tipo de escola e prática de atividade física. Para a avaliação da renda familiar foi questionada a renda de todos os moradores do domicilio e o número de pessoas dependentes da renda declarada, a fim de se realizar o cálculo da renda per capita. Para a classificação dos indivíduos, considerou-se a mediana da renda per capita.

\section{Consumo alimentar}

O consumo alimentar foi avaliado por três recordatórios de 24 horas aplicados por nutricionista, em dias não consecutivos, sendo uma das avaliações referente ao fim de semana. As crianças responderam ao inquérito alimentar acompanhadas de seus pais ou responsáveis, sendo entrevistado preferencialmente aquele diretamente envolvido com a alimentação da criança.

Para auxiliar os participantes na determinação do tamanho das porções ingeridas, foram utilizados utensílios caseiros e figuras de porcionamento de alguns alimentos em álbum fotográfico ${ }^{16}$. A análise dos dados dietéticos foi realizada com o auxílio do software Diet Pro $^{\circledR}$ 5i, versão 5.8.para quantificar o consumo de energia ${ }^{17}$. Os alimentos foram agrupados e classificados como marcadores de alimentação "saudável" e "não saudável”. Para essa classificação levou-se em consideração as recomendações do Guia Alimentar para população Brasileira ${ }^{18}$ que estabelece o consumo de alimentos in natura ou minimamente processados (marcadores da alimentação "saudável") e desencoraja o consumo de produtos ultraprocessados (marcadores da alimentação “não saudável”) (Quadro 1).

Foram considerados produtos ultraprocessados as formulações industriais feitas com cinco ou mais ingredientes, tais como aditivos, antioxidantes, estabilizantes e conservantes ${ }^{19}$. Como não há recomendações referentes ao consumo de UPP, este foi considerado "regular" quando inferior ao percentil $75 \mathrm{da}$ amostra, enquanto que, para o consumo de alimentos marcadores da alimentação saudável, adotou-se como "regular" aqueles com ingestão superior ao percentil 75 .

\section{Análise de dados}

Para análise dos perfis de consumo alimentar infantil foi utilizada a técnica Two-Step Cluster (TSC), realizado no software Stata versão 13.0. Este método permite agrupar a amostra em perfis de indivíduos com consumo alimentar semelhantes entre si. Após a constituição dos grupos, verificou-se a associação dos mesmos com as variáveis sociodemográficas.

Para comparação do consumo dos grupos alimentares entre os grupos formados foi utilizado o Teste T de Student. A análise bivariada foi efetuada a partir de modelos de regressão de Poisson com variância robusta, tendo como variável dependente os grupos formados e como variáveis explicativas os hábitos alimentares e aspectos socioeconômicos. Foi realizado o cálculo da Razão de Prevalência (RP) com intervalo de confiança de 95\% (IC 95\%) e para todas as análises, foi adotado o valor de significância de 5\%.

\section{Resultados}

Observou-se que $52,1 \%(\mathrm{n}=197)$ das crianças eram do sexo feminino, 50,3\% $(\mathrm{n}=190)$ tinham 9 anos de idade, $68,5 \%(\mathrm{n}=259)$ eram não brancos e 70,9\% ( $n=268)$ eram de escolas públicas.

O perfil alimentar dos indivíduos foi analisado em dois grupos: "saudável" e "não saudável". O grupo "saudável" foi composto por 116 crianças $(30,7 \%)$, representando menos da metade das crianças da amostra (Tabela 1).

Observou-se a presença de arroz e feijão, verdura, legumes, leite, frutas e carnes nos dois grupos. No entanto, entre os indicadores da alimentação não saudável, destaca-se a presença de ultraprocessados (fast foods, biscoitos e embutidos), responsáveis por diferenciar os perfis "saudável" " "não saudável" (Tabela 1).

A medida de contribuição de cada variável para a formação dos perfis se dá pelo consumo regular ou irregular dos grupos alimentares (de acordo com o percentil 75). Alguns grupos (condimentos, bebidas industrializadas, macarrão 
Quadro 1. Classificação dos alimentos em grupos de alimentação saudável e não saudável.

\begin{tabular}{|c|c|c|}
\hline \multirow{6}{*}{$\begin{array}{l}\text { Indicadores da } \\
\text { Alimentação } \\
\text { saudável }\end{array}$} & Grupos Alimentares & Alimentos \\
\hline & Leite & Leite desnatado, integral, sem lactose e em pó. \\
\hline & Arroz e feijão & Arroz branco, integral e feijão \\
\hline & Carnes e ovos & Carnes de boi, porco e frango cozidos, ovo de galinha cozido. \\
\hline & \multicolumn{2}{|l|}{ Verduras e legumes } \\
\hline & Frutas & \\
\hline \multirow{7}{*}{$\begin{array}{l}\text { Indicadores da } \\
\text { Alimentação não- } \\
\text { saudável }\end{array}$} & Embutidos & Linguiça, salsicha, presunto, salame e mortadela. \\
\hline & Lanches tipo fast food & $\begin{array}{l}\text { Cachorro-quente, hambúrguer, pizza, lasanha, salgados fritos e } \\
\text { misto quente. }\end{array}$ \\
\hline & Açúcares e doces & $\begin{array}{l}\text { Açúcar de adição, achocolatados, balas, pirulitos, chocolate, } \\
\text { sobremesas, sorvete e milkshake. }\end{array}$ \\
\hline & Bebidas industrializadas & Sucos artificiais, refrigerantes e bebidas achocolatadas. \\
\hline & Biscoitos & Biscoitos doces, biscoito recheado e salgadinho tipo chips. \\
\hline & Condimentos & Maionese, mostarda, ketchup, molho inglês e molho de tomate. \\
\hline & Massas & Macarrão instantâneo \\
\hline
\end{tabular}

Tabela 1. Formação dos grupos alimentares das crianças. Viçosa, MG, 2015.

\begin{tabular}{|c|c|}
\hline $\begin{array}{c}\text { Cluster "Saudável" } \\
\text { n (\%) } \\
116(30,7) \\
\end{array}$ & $\begin{array}{c}\text { Cluster "Não saudável" } \\
\text { n (\%) } \\
262(69,3) \\
\end{array}$ \\
\hline Arroz e feijão & Fast-foods \\
\hline Verduras e legumes & Biscoito \\
\hline Leite & Embutidos \\
\hline Frutas & Arroz e feijão \\
\hline \multirow[t]{4}{*}{ Carnes } & Verduras e legumes \\
\hline & Leite \\
\hline & Frutas \\
\hline & Carnes \\
\hline
\end{tabular}

Análise Two Step Cluster

Tabela 2. Consumo médio dos grupos alimentares em cada cluster pelas crianças. Viçosa, MG, 2015.

\begin{tabular}{lccc}
\hline $\begin{array}{c}\text { Grupos } \\
\text { Alimentares } \\
\text { (g/dia) }\end{array}$ & $\begin{array}{c}\text { Cluster } \\
\text { “Saudável” } \\
\mathbf{1 1 6}(\mathbf{3 0 , 7 \% )})\end{array}$ & $\begin{array}{c}\text { Cluster } \\
\text { "Não } \\
\text { saudável” } \\
\mathbf{2 6 2} \mathbf{( 6 9 , 3 \% )}\end{array}$ & $\begin{array}{c}\text { Valor } \\
\text { de P }\end{array}$ \\
\hline Arroz e feijão & 203,9 & 193,4 & 0,385 \\
Verduras e & 74,3 & 41,9 & $<0,001$ \\
Legumes & & & \\
Leite & 159,6 & 132,7 & 0,036 \\
Frutas & 83,1 & 64,9 & 0,084 \\
Carnes & 35,3 & 31,3 & 0,286 \\
Fast-food & 25,5 & 58,8 & $<0,001$ \\
Biscoito & 84,0 & 109,5 & $<0,001$ \\
Embutidos & 12,0 & 20,2 & $<0,001$ \\
\hline Teste T de Student. & & &
\end{tabular}

instantâneo e doces) tiveram um consumo semelhante em todos os clusters, logo os mesmos não foram responsáveis por diferenciá-los e por isso ao fim da análise estatística não foram incluídos nos grupos alimentares formados.

Verificou-se que o grupo "saudável” apresentou maior consumo de verduras e legumes e leite enquanto que as crianças do grupo "não saudável" tiveram maior consumo de fast foods, biscoito e embutidos (Tabela 2).

Ao avaliar a ingestão calórica dos UPP consumidos em cada grupo, a contribuição energética no grupo "saudável” (20,5\%) foi menor que no grupo "não saudável” $(24,1 \%)(\mathrm{p}=0,043)$.

A análise univariada mostrou associação entre as variáveis sociodemográficas e ambientais com o perfil alimentar infantil. Observou-se que o consumo "não saudável" foi maior nas crianças de escolas privadas ( $\mathrm{RP}=1,28(1,20-1,37)$, $\mathrm{p}=<0,001)$, que sempre levam lanche para a escola $(R P=1,13(1,01-1,27), p=0,022)$, que não recebiam Bolsa Família $(\mathrm{RP}=1,22(1,10$ $1,36), \mathrm{p}=<0,001)$, com maior renda $(\mathrm{RP}=1,13$ $(1,05-1,23), \mathrm{p}=0,001)$ e que a mãe trabalha fora $(\mathrm{RP}=1,26(1,21-1,31), \mathrm{p}=<0,001)$. Em contrapartida, crianças não brancas $(\mathrm{RP}=0,91(0,84-0,99)$, $\mathrm{p}=0,031)$ e que não praticavam atividade física $(\mathrm{RP}=0,91 \quad(0,84-0,98), \mathrm{p}=0,026)$ apresentaram menor consumo do grupo "não saudável" (Tabela 3). Após o ajuste no modelo multivariado, observou-se que crianças de escola privada, que não recebiam bolsa família e que a mãe trabalhava fora, tiveram maior consumo dos alimentos do grupo "não saudável” (Tabela 4). 
Tabela 3. Análise univariada de variáveis exploratórias e associação com os clusters como variável dependente. Viçosa, MG, 2015.

\begin{tabular}{|c|c|c|c|c|}
\hline \multirow{2}{*}{ Variável } & \multicolumn{2}{|c|}{ Grupo Saudável } & \multicolumn{2}{|c|}{ Grupo Não Saudável } \\
\hline & Referência & Valor de $\mathbf{P}$ & RP/ IC (95\%) & Valor de $\mathrm{P}$ \\
\hline \multicolumn{5}{|l|}{ Etnia } \\
\hline Branco & 1,0 & - & 1,0 & - \\
\hline Não Branco & 1,0 & - & $0,91(0,84-0,99)$ & 0,031 \\
\hline \multicolumn{5}{|l|}{ Sexo } \\
\hline Masculino & 1,0 & - & 1,0 & - \\
\hline Feminino & 1,0 & - & $1,04(0,97-1,13)$ & 0,225 \\
\hline \multicolumn{5}{|l|}{ Escola } \\
\hline Pública & 1,0 & - & 1,0 & - \\
\hline Privada & 1,0 & - & $1,28(1,20-1,37)$ & $<0,001$ \\
\hline \multicolumn{5}{|c|}{ Leva lanche para a escola } \\
\hline Nunca & 1,0 & - & 1,00 & - \\
\hline Ás vezes & 1,0 & - & $0,89(0,79-1,01)$ & 0,085 \\
\hline Sempre & 1,0 & - & $1,13(1,01-1,27)$ & 0,022 \\
\hline \multicolumn{5}{|l|}{ Pratica atividade física } \\
\hline Sim & 1,0 & - & 1,00 & - \\
\hline Não & 1,0 & - & $0,91(0,84-0,98)$ & 0,026 \\
\hline \multicolumn{5}{|l|}{ Recebe bolsa família } \\
\hline $\operatorname{Sim}$ & 1,0 & - & 1,00 & - \\
\hline Não & 1,0 & - & $1,22(1,10-1,36)$ & $<0,001$ \\
\hline \multicolumn{5}{|l|}{ Renda per capita } \\
\hline$<500,00$ & 1,0 & - & 1,00 & - \\
\hline$>=500,00$ & 1,0 & - & $1,13(1,05-1,23)$ & 0,001 \\
\hline \multicolumn{5}{|l|}{ Escolaridade materna } \\
\hline$<9$ anos & 1,0 & - & 1,00 & - \\
\hline$\geq 9$ anos & 1,0 & - & $1,08(0,99-1,18)$ & 0,065 \\
\hline \multicolumn{5}{|l|}{ Escolaridade paterna } \\
\hline$<9$ anos & 1,0 & - & 1,00 & - \\
\hline$\geq 9$ anos & 1,0 & - & $1,08(0,99-1,17)$ & 0,059 \\
\hline \multicolumn{5}{|l|}{ Mãe trabalha fora } \\
\hline Não & 1,0 & - & 1,00 & - \\
\hline Sim & 1,0 & - & $1,26(1,21-1,31)$ & $<0,001$ \\
\hline
\end{tabular}

Tabela 4. Modelo de regressão multivariado com os clusters como variável dependente. Viçosa, MG, 2015.

\begin{tabular}{lcccc}
\hline \multicolumn{1}{c}{ Variável } & \multicolumn{2}{c}{ Grupo Saudável } & \multicolumn{2}{c}{ Grupo Não Saudável } \\
& Referência & Valor de P & RP/ IC (95\%) & Valor de P \\
\hline Escola & 1,0 & - & 1,0 & - \\
$\quad \begin{array}{l}\text { Pública } \\
\text { Privada }\end{array}$ & 1,0 & - & $1,25(1,15-1,35)$ & $<0,001$ \\
Recebe Bolsa Família & & & & - \\
$\quad$ Sim & 1,0 & - & 1,0 & 0,036 \\
$\quad$ Não & 1,0 & - & $1,13(1,01-1,26)$ & - \\
Mãe trabalha fora & & & 1,0 & $<0,001$ \\
$\quad$ Não & 1,0 & - & $1,38(1,28-1,49)$ & - \\
$\quad$ Sim & 1,0 & - & 1,0 & 0,992 \\
Pratica atividade física & & & - &
\end{tabular}




\section{Discussão}

Neste estudo, foram encontrados dois perfis (clusters) de consumo alimentar, sendo que a ingestão de fast foods, biscoitos e embutidos diferenciou os perfis "saudável" e "não saudável".

O grupo alimentar "saudável" apresentou menor prevalência na amostra $(30,7 \%)$, refletindo a realidade brasileira encontrada em outro estudo, no qual apenas $9 \%$ das crianças brasileiras atingiram as porções recomendadas para frutas e verduras $^{20}$. Neste trabalho, não houve diferença entre os grupos quanto à ingestão de frutas, pois em ambos o consumo foi abaixo do recomendado. No entanto, tais alimentos são indispensáveis para a saúde por serem fontes de vitaminas e minerais, além da prevenção do risco de doenças crônicas ${ }^{21,22}$.

Os fast foods, biscoitos e embutidos consumidos por crianças no grupo "não saudável" se caracterizam como pouco nutritivos e de alta densidade energética ${ }^{3}$. Atualmente, observa-se um grande apelo publicitário em relação a esse grupo de alimentos, o que leva a um consumo cada vez mais frequente entre o público infantil ${ }^{23}$. Esse maior consumo pode predispor as crianças ao excesso de peso e à hipertensão $\operatorname{arterial}^{24}$.

Segundo dados da POF (2008-2009), os UPP contribuíam com $28 \%$ da ingestão energética diária ${ }^{7}$. Tal resultado é próximo ao encontrado neste estudo, no qual os UPP contribuíram com $20,5 \%$ no grupo "saudável" e $24,1 \%$ no grupo "não saudável" e vai ao encontro a outros estudos que avaliaram o consumo de ultraprocessados por crianças, que identificaram uma contribuição de 19,7 a 47,0\% deste grupo na ingestão calórica total ${ }^{12,14,24}$.

Ressalta-se que o consumo de produtos processados tornou-se um hábito desde os primeiros anos de vida, com a introdução da alimentação complementar ${ }^{2}$. No Brasil, uma a cada três crianças menores de dois anos já consumiu refrigerante e $60,8 \%$ consumiu biscoitos ou bolos ${ }^{25}$. Entre os escolares esse cenário não é diferente. Estudo realizado no Maranhão encontrou um consumo de refrigerante maior que o de sucos naturais. Ao avaliar esse consumo entre as escolas, a ingestão na rede privada foi significativamente maior que na pública ${ }^{26}$.

Acredita-se que esse elevado consumo pelo grupo infantil seja devido aos estabelecimentos em torno das escolas que favorecem o consumo de produtos UPP. Em Santos (SP), os pontos de venda que ofertavam UPP estavam significativamente mais próximos das escolas em relação aos que comercializavam alimentos in natura e minimamente processados ${ }^{27}$. Ademais a publicidade dos produtos alimentares tem sido cada vez mais voltada para o incentivo do consumo de UPP, com enfoque nos benefícios dos fortificados. Tais questões levam o consumidor a acreditar que produtos industrializados fortificados se caracterizam como saudáveis. Acredita-se que a exposição por apenas 30 segundos a comerciais de alimentos é capaz de influenciar a escolha de crianças a determinado produto ${ }^{28}$.

Neste estudo, o consumo "não saudável" foi maior entre as crianças matriculadas em escolas privadas. Sabe-se que neste caso, os estudantes tendem a consumir lanches trazidos de casa ou comprados na cantina da escola. Um estudo realizado no Rio de Janeiro evidenciou que estes lanches normalmente são compostos por alimentos de alta densidade energética ${ }^{29}$. Além disso, nas escolas públicas tem-se a atuação do Programa Nacional de Alimentação Escolar (PNAE), que tem como objetivo promover a alimentação saudável ${ }^{30}$. Estudo realizado na Paraíba, avaliou o índice estatura/idade e identificou maior vulnerabilidade nutricional das crianças que não consumiam a merenda escolar ${ }^{31}$.

Filhos de mães que trabalham fora tiveram maior prevalência de consumo de alimentos do grupo "não saudável". É importante ressaltar que os produtos UPP se apresentaram como atrativos para a população, devido a sua praticidade, uma vez que não necessitam de quase nenhuma preparação culinária, sendo seu uso intensificado com a maior participação da mulher no mercado de trabalho e o estilo de vida contemporâneo, caracterizado pela falta de tempo para o preparo das refeições ${ }^{3}$.

Segundo dados da POF (2008-2009), 28\% do gasto com alimentação era atribuído à compra de alimentos para consumo fora de casa, sendo parte deste constituído por UPP ${ }^{7}$. Entretanto, essa mudança no perfil alimentar não é algo restrito à população brasileira, estudos recentes demonstraram que consiste em um fenômeno de consumo, caracterizado pelo surgimento de indústrias transnacionais de alimentos, acompanhado da redução do preço relativo desses produtos ${ }^{3,32}$. No Canadá, a participação dos UPP na alimentação da população aumentou de $24,4 \%$ para $54,9 \%$ entre os anos de 1938-1939 a 200132.

O consumo "não saudável" foi mais prevalente entre as crianças cujas famílias não recebiam Bolsa Família. Acredita-se que as famílias beneficiadas pelo programa utilizam grande parte do recurso na aquisição de alimentos saudáveis, 
o que contribui para uma melhora na qualidade e quantidade da alimentação das famílias ${ }^{33}$. Ademais, estudo realizado por Pedraza et al. ${ }^{34}$ observou resultado positivo quanto à efetividade do programa no que se refere à recuperação e manutenção do estado nutricional infantil.

Ressalta-se ainda que, para o recebimento do benefício é preciso cumprir algumas condicionalidades, entre elas acompanhamento periódico do estado nutricional e de saúde das famílias; participação em ações de educação alimentar e nutricional; e frequência e assiduidade das crianças na escola ${ }^{35}$. Essa assiduidade das crianças garante às mesmas o recebimento da merenda escolar, e como já colocado, trata-se de uma alimentação adequada nutricionalmente.

Alguns pontos positivos deste trabalho devem ser salientados. Este é um dos poucos estudos realizados em países em desenvolvimento que investigou os fatores associados ao consumo de alimentos ultraprocessados na infância, sendo o primeiro estudo brasileiro de base-populacional com crianças pré-púberes. Como há relação entre o consumo de ultraprocessados e o aumen-

\section{Colaboradores}

MA Silva: coleta e análise dos dados, elaboração do artigo. LC Milagres: coleta e análise dos dados, elaboração do artigo. APP Castro: coleta e análise dos dados, elaboração do artigo. MDS Filgueiras: coleta e análise dos dados, elaboração do artigo. NP Rocha: coleta e análise dos dados, elaboração do artigo. HHM Hermsdorff: elaboração do artigo e orientação do trabalho. GZ Longo: elaboração do artigo e orientação do trabalho. JF Novaes: elaboração do artigo e orientação do trabalho. to do sobrepeso/obesidade ${ }^{36}$ esta é uma fase importante para avaliar os fatores associados a este consumo, uma vez que a infância é o período de formação dos hábitos alimentares, e todo esforço deve ser garantido para a manutenção destes em fases posteriores da vida. Estes achados são consistentes com outros estudos, sugerindo que o consumo de ultraprocessados tem aumentado. Como limitação deste estudo, destaca-se a ausência de algumas informações nas tabelas de composição de alimentos, principalmente em relação aos produtos ultraprocessados, uma vez que a cada dia surgem novos produtos no mercado.

Conclui-se que o consumo de produtos ultraprocessados esteve associado às melhores condições socioeconômicas das famílias das crianças. Esses resultados ressaltam a importância de adotar medidas preventivas, com ênfase na redução do consumo de produtos ultraprocessados, por meio de ações de educação alimentar e nutricional com pais e professores, para melhoria das condições de vida das crianças e suas famílias, bem como o acesso às informações para a compra e consumo de alimentos saudáveis.

\section{Referências}

1. Monteiro CA, Levy RB, Claro RM, Castro IRR, Cannon G. Increasing consumption of ultra-processed foods and likely impact on human health: evidence from Brazil. Public Health Nutrition 2010;14(1):5-13.

2. Barcelos GT, Rauber F, Vitolo MR. Produtos processados e ultraprocessados e ingestão de nutrientes em crianças. Ciência \& Saúde 2014;7(3):155-161.

3. Martins APB, Levy RB, Claro RM, Moubarac JC, Monteiro CA. Participação crescente de produtos ultraprocessados na dieta brasileira (1987-2009). Rev Saude Publ 2013;47(4):656-665.

4. Louzada MLC, Martins APB, Canella DS, Baraldi LG, Levy RB, Claro RM, Moubarac JC, Cannon G, Monteiro CA. Alimentos ultraprocessados e perfil nutricional da dieta no Brasil. Rev Saude Publ 2015;49(38):1-11.

5. Instituto Brasileiro de Geografia e Estatística (IBGE). Pesquisa Nacional de Saúde do Escolar. Brasília: IBGE; 2012.

6. Bielemann RM, Motta JVS, Minten GC, Horta BL Gigante DP. Consumo de alimentos ultraprocessados e impacto na dieta de adultos jovens. Rev Saude Publ 2015;49(28):1-10.

7. Instituto Brasileiro de Geografia e Estatística (IBGE). Pesquisa de orçamentos familiares 2008-2009: avaliação nutricional da disponibilidade de alimentos no Brasil. Rio de Janeiro: IBGE; 2010.

8. Bertuol CD, Navarro AC. Consumo alimentar e prevalência de sobrepeso/obesidade em pré-escolares de uma escola infantil pública. Revista Brasileira de Obesidade, Nutrição e Emagrecimento 2015;9(52):127-134. 
9. Martins APB, Levy RB, Claro RM, Moubarac JC, Monteiro CA. Participação crescente de produtos ultraprocessados na dieta brasileira (1987-2009). Rev Saude Publ 2013;47(4):656-665.

10. Wijtzes AI, Jansen W, Jansen PW, Jaddoe VW, Hofman A, Raat H. Maternal educational level and preschool children's consumption of high-calorie snacks and sugar-containing beverages: mediation by the family food environment. Prev Med 2013;57(5):607-612.

11. Momm N, Hofelmann DA. Qualidade da dieta e fatores associados em crianças matriculadas em uma escola municipal de Itajaí, Santa Catarina. Cad Saude Colet 2014;22(1):32-39.

12. Karnopp EVN, Vaz JS, Schafer AA, Muniz LC, Souza RLV, Santos I, Gigante DP, Assunção MCF. Food consumption of children younger than 6 years according to the degree of food processing. J Pediatr 2017;93(1):70-78.

13. Bielemann RM, Motta JVS, Minten GC, Horta BL, Gigante DP. Consumo de alimentos ultraprocessados e impacto na dieta de adultos jovens. Rev Saude Publ 2015;49(28):1-10.

14. Sparrenberger K, Friedrich RR, Schiffner MD, Schuch I, Wagner MB. Ultra-processed food consumption in children from a Basic Health Unit. J Pediatr 2015;91(6):535-542.

15. Luiz RR, Magnanini MMF. O tamanho da amostra em investigações epidemiológicas. In: Medronho RA, Bloch KV, Luiz RR, Werneck GL. Epidemiologia. $2^{\text {a }}$ Edição. São Paulo: Atheneu; 2009. p. 415-429.

16. Zabotto CB, Vianna RPT, Gil MF. Registro fotográfico para inquéritos dietéticos: utensílios e porções. Goiânia: Nepa-Unicamp; 1996.

17. DIET, PRO. "VERSÃO 5. i.”. Software de avaliação nutricional e prescrição dietética. UFV, Viçosa, MG, Brasil. AS Sistema; 1997.

18. Brasil. Ministério da Saúde, Secretaria de Atenção à Saúde, Departamento de Atenção Básica, Coordenação Geral de Alimentação e Nutrição. Guia alimentar para a população brasileira (versão para consulta pública). Brasília: MS; 2014.

19. Monteiro CA, Cannon G, Levy R, Moubarac JC, Jaime P, Martins AP, Canella D, Louzada M, Parra D. NOVA. A estrela brilha. Word Nutrition 2016;7(1-3):28-40.

20. Chafee BW. Early life factors among the many influences of child fruit and vegetable consumption. $J P e$ diatr 2014;90(5):437-439.

21. Castanho GKF, Marsola FC, Mclellan KCP, Nicola M, Moreto F, Burini RC. Consumo de frutas, verduras e legumes associados à Síndrome Metabólica e seus componentes em amostra populacional adulta. Ciên Saude Colet 2013;18(2):385-392.

22. Muniz LC, Zanini RV, Schneider BC, Tassitano RM, Feitosa WMN, Gonzáles-Chica DA. Prevalência e fatores associados ao consumo de frutas, legumes e verduras entre adolescentes de escolas públicas de Caruaru, PE. Ciên Saude Colet 2013;18(2):393-404.

23. Porto RB, Oliveira-Castro JM. Say - do correspondence in brand choice: Interaction effects of past and current contingencies. The Psychological Record 2013;63(2):345-362.
24. Payab M, Kelishad R, Qorbani M, Motlagh ME, Ranjbar SH, Ardalan G, Zahedi H, Chinian M, Asayesh $\mathrm{H}$, Larijani B, Heshmat R. Association of junk food consumption with high blood pressure and obesity in Iranian children and adolescents: the Caspian-IV study. J Pediatr 2015;91(2):196-205.

25. Jaime PC, Frias PG, Monteiro HOC, Almeida PVB, Malta DC. Assistência em saúde e alimentação não saudável em crianças menores de dois anos: dados da Pesquisa Nacional de Saúde, Brasil, 2013. Rev Bras Saude Mater Infant 2016;16(2):159-167.

26. Conceição SIO, Santos CJN, Silva AAM, Silva JS, Oliveira TC. Consumo alimentar de escolares das redes pública e privada de ensino em São Luís, Maranhão. Rev Nutr 2010;23(6):993-1004.

27. Leite FHM, Oliveira MA, Cremm EC, Abreu DSC, Maron LR, Martins PA. Availability of processed foods in the perimeter of public schools in urban areas. $J$ Pediatr 2012;88(4):328-334.

28. Momm D, Hofelmann DA. Qualidade da dieta e fatores associados em crianças matriculadas em uma escola municipal de Itajaí, Santa Catarina. Cad Saude Colet 2014;22(1):32-39.

29. Barros MS, Fonseca VM, Meio MDBB, Chaves CR. Excesso de peso entre adolescentes em zona rural e a alimentação escolar oferecida. Cad Saude Colet 2013;21(2):201-208.

30. Libermann AP, Bertolini GRF. Tendências de pesquisa em políticas públicas: uma avaliação do Programa Nacional de Alimentação Escolar - PNAE. Ciên Saude Colet 2015;20(11): 3533-3546.

31. Pedraza DF, Silva FA, Melo NLS, Araujo EMN, Souza CPC. Estado nutricional e hábitos alimentares de escolares de Campina Grande, Paraíba, Brasil. Ciên Saude Colet 2017;22(2):469-477.

32. Moubarac JC, Claro KM, Baraldi LG, Levy RB, Martins AP, Cannon G, Monteiro CA. International diferences in cost and consumption of ready to consume food and drink products: United Kingdom and Brazil 2008-2009. Global Public Health 2013;8(7):845-856.

33. Vasconcelos PN, Cavalcanti DS, Leal LP, Osório MM, Fialho MB. Tendência temporal e fatores determinantes da anemia em crianças de duas faixas etárias (6-23 e 24-59 meses) no Estado de Pernambuco, Brasil, 1997-2006. Cad Saude Publica 2014;30(8):17771787.

34. Pedraza DF, Rocha ACD, Sousa CPC. Crescimento e deficiências de micronutrientes: perfil das crianças assistidas no núcleo de creches do governo da Paraíba, Brasil. Ciên Saude Colet 2013;18(11):3379-3390.

35. Wolf MR, Barros Filho AA. Estado nutricional de beneficiários do programa Bolsa Família no Brasil - um revisão sistemática. Ciên Saude Colet 2014;19(5):13311338.

36. Mendonça RD, Pimenta AM, Gea A, Fuente-Arrillaga C, Martinez-Gonzales MA, Lopes ACS, Bes-Rastrollo M. Ultraprocessed food consumption and risk of overweight and obesity: the University of Navarra Follow-up (SUN) cohort study. Am J Clin Nutr 2016;104(5):1433-1440.

Artigo apresentado em 29/05/2017

Aprovado em 17/04/2018

Versão final apresentada em 19/04/2018 